\title{
A Subband-Selective Broadband GSC With Cosine-Modulated Blocking Matrix
}

\author{
Wei Liu, Member, IEEE, Stephan Weiss, Member, IEEE, and Lajos Hanzo, Fellow, IEEE
}

\begin{abstract}
A novel subband-selective generalized sidelobe canceller (GSC) for partially adaptive broadband beamforming is proposed. The columns of the blocking matrix are derived from a prototype vector by cosine modulation, and the broadside constraint is incorporated by imposing zeros on the prototype vector appropriately. These columns constitute a series of bandpass filters, which select signals with specific directions of arrival and frequencies. This results in a high-pass-type bandlimited spectra of the blocking matrix outputs, which is further exploited by subband decomposition and suitably discarding the low-pass subbands prior to running independent unconstrained adaptive filters in each nonredundant subband. By these steps, the computational complexity of a GSC implementation is greatly reduced compared to fully adaptive GSC schemes, while performance is comparable or even enhanced due to subband decorrelation in both spatial and temporal domains.
\end{abstract}

Index Terms-Generalized sidelobe canceller (GSC).

\section{INTRODUCTION}

A DAPTIVE broadband beamforming has found many applications in various areas ranging from sonar and radar to wireless communications [1]. A general beamformer with $M$ sensors receiving a signal of interest from the direction of arrival (DOA) $\theta$ is shown in Fig. 1. In broadband beamforming, to achieve high interference rejection and spatial resolution, arrays with a large number of sensors $M$ and filter coefficients $J$ have to be employed, resulting in a considerable computational burden. To reduce the computational complexity, partially adaptive beamforming [2]-[5] is an option, which employs only a subset of the available degrees of freedom (DOFs) in the filter update process at the expense of a somewhat reduced performance. Recently, subband methods were also introduced [6]-[8] for broadband beamforming with low computational complexity, where subband adaptive filters with reduced update rate and filter order are employed in the adaptive process of beamforming. Because of the prewhitening effect, subband methods can also achieve a faster convergence speed for least mean square (LMS)-type adaptive algorithms than their full-band counterparts.

In this paper, we combine partial adaptivity and subband processing methods and propose a partially adaptive generalized sidelobe canceller (GSC) [9] with a novel subband-selective cosine-modulated blocking matrix. In this structure, the column

Manuscript received December 3, 2002; revised May 6, 2003.

The authors are with the Communications Research Group, Department of Electronics and Computer Science, University of Southampton, Southampton SO17 1BJ, U.K. (e-mail: w.liu@ecs.soton.ac.uk; s.weiss@ecs.soton.ac.uk; 1.hanzo@ecs.soton.ac.uk).

Digital Object Identifier 10.1109/TAP.2004.825096

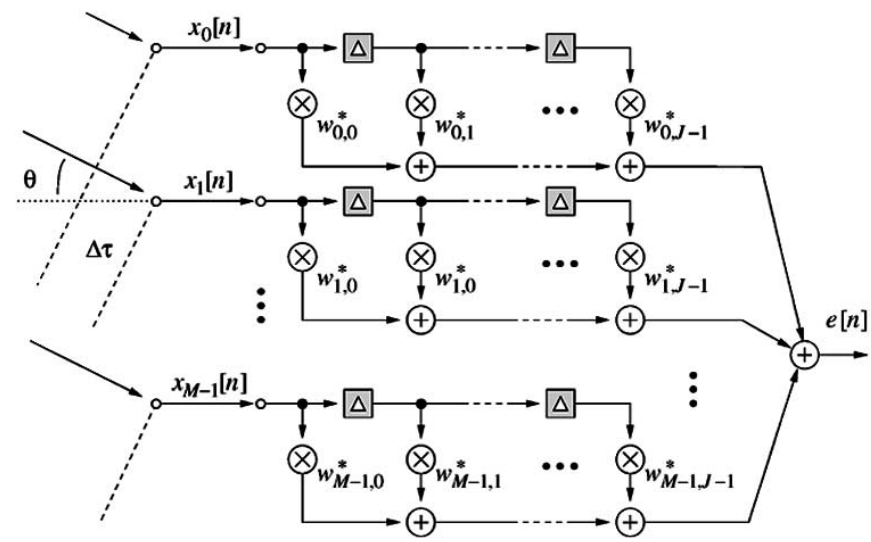

Fig. 1. Signal impinging from an angle $\theta$ onto a broadband beamformer with $M$ sensors, each followed by a $J$-tap filter.

vectors of the blocking matrix of reduced dimension constitute a series of cosine-modulated bandpass filters, which separate the impinging signals and interference into components of different DOAs and frequencies. This results in bandlimited spectra of the blocking matrix output and is further exploited by subband decomposition and appropriately discarding the low-pass subbands prior to invoking independent unconstrained adaptive filters in each nonredundant subband. By these steps, the computational complexity of the system is greatly reduced. Additionally, a faster convergence speed will be achieved by joint spatial and spectral decorrelation. An advantage of this method is that the subbands discarded during the adaptation can be determined a priori and are independent of the signal environment. A further reduction of the computational complexity can be potentially achieved by monitoring the remaining subbands and dynamically discarding those subbands whose signal power falls below a given threshold [5].

This paper is organized as follows. Section II briefly reviews GSC-based fully and partially adaptive broadband beamforming prior to proposing a special subband-selective GSC. For this scheme, a novel cosine-modulated blocking matrix is introduced in Section III. Finally, simulations underlining the benefit of our proposed method are discussed in Section IV, and conclusions are drawn in Section V.

\section{SubBand-Selective Generalized Sidelobe CANCELLER}

A. GSC

A linearly constrained minimum variance (LCMV) beamformer [10] performs the minimization of the variance or power of the output signal $e[n]$ of the structure in Fig. 1 with respect to 
some given spatial and spectral constraints. For a beamformer with $M$ sensors and $J$ filter taps following each sensor, the output $e[n]$ can be expressed as

$$
e[n]=\boldsymbol{w}^{H} \boldsymbol{x}
$$

with

$$
\begin{aligned}
\boldsymbol{w} & =\left[\begin{array}{llll}
\boldsymbol{w}_{0}^{T} & \boldsymbol{w}_{1}^{T} & \cdots & \boldsymbol{w}_{J-1}^{T}
\end{array}\right]^{T} \\
\boldsymbol{w}_{j} & =\left[\begin{array}{llll}
w_{0, j} & w_{1, j} & \cdots & w_{M-1, j}
\end{array}\right]^{T} \\
\boldsymbol{x} & =\left[\begin{array}{llll}
\boldsymbol{x}^{T}[n] & \boldsymbol{x}^{T}[n-1] & \cdots & \boldsymbol{x}^{T}[n-J+1]
\end{array}\right]^{T} \\
\boldsymbol{x}[n-j] & =\left[\begin{array}{llll}
x_{0}[n-j] & x_{1}[n-j] & \cdots & x_{M-1}[n-j]
\end{array}\right]^{T}
\end{aligned}
$$

where $\{\cdot\}^{T}$ and $\{\cdot\}^{H}$ denote transpose and Hermitian transpose operations, respectively. Each vector $\boldsymbol{w}_{j}, j=0, \ldots, J-1$, contains the $M$ complex conjugate coefficients found at the $j$ th tap position of the $M$ attached filters, and $x[n-j], j=0, \ldots, J-1$ holds the $j$ th data slice in the array corresponding to the $j$ th coefficient vector $\boldsymbol{w}_{j}$.

The LCMV problem can be formulated as

$$
\min _{\boldsymbol{w}} \boldsymbol{w}^{H} \boldsymbol{R}_{x x} \boldsymbol{w} \quad \text { subject to } \boldsymbol{C}^{H} \boldsymbol{w}=\boldsymbol{f}
$$

where $\boldsymbol{R}_{x x}$ is the covariance matrix of the received array data in $\boldsymbol{x}, \boldsymbol{C}$ the $M J \times J$ constraint matrix, and $\boldsymbol{f}$ the $J \times 1$ response vector. If the signal of interest arrives from broadside, then $C$ has the form

$$
\boldsymbol{C}=\left[\begin{array}{lll}
\mathbf{1}_{M} & & \mathbf{0} \\
& \ddots & \\
\mathbf{0} & & \mathbf{1}_{M}
\end{array}\right]
$$

and $\boldsymbol{f}$ can be $[1,0, \ldots, 0]^{T}$, where $1_{M}$ is an $M \times 1$ vector with all its elements being equal to unity. A convenient way of solving the constrained optimization in (6) is by using a GSC [9], which splits the constrained optimization problem into two subspaces, one fullfilling the constraints by a quiescent vector $\boldsymbol{w}_{q}$, the other one orthognal to the constraints by a blocking matrix $\boldsymbol{B}$. Thereafter, standard unconstrained optimization algorithms such as LMS or recursive least squares (RLS) algorithms can be invoked [11].

Fig. 2 shows the principle of a GSC [9], [12], where the upper branch signal $d[n]$ is obtained via $\boldsymbol{w}_{q}$

$$
d[n]=\boldsymbol{w}_{q}^{H} \cdot \boldsymbol{x} \quad \text { with } \quad \boldsymbol{w}_{q}=\boldsymbol{C}\left(\boldsymbol{C}^{H} \boldsymbol{C}\right)^{-1} \boldsymbol{f} .
$$

Assume that we have $\boldsymbol{B} \in \mathbb{C}^{M \times L}$, which is composed of $L$ linearly independent column vectors

$$
\begin{aligned}
\boldsymbol{B} & =\left[\begin{array}{llll}
\boldsymbol{b}_{0} & \boldsymbol{b}_{1} & \ldots & \boldsymbol{b}_{L-1}
\end{array}\right] \\
\boldsymbol{b}_{l} & =\left[\begin{array}{llll}
b_{l}[0] & b_{l}[1] & \ldots & b_{l}[M-1
\end{array}\right]^{H}
\end{aligned}
$$

with $l=0, \ldots, L-1$. The multichannel input signal $\boldsymbol{u}[n]=\left[\begin{array}{llll}u_{0}[n] & u_{1}[n] & \ldots & u_{L-1}[n]\end{array}\right]^{T}$ of the following multichannel adaptive filtering (MCAF) process is obtained by $\boldsymbol{u}[n]=\boldsymbol{B}^{H} \boldsymbol{x}[n]$, whereby the blocking matrix must satisfy

$$
\mathbf{1}^{H} \mathbf{B}=\mathbf{0}
$$

with $C$ given in (7) and 1 being an $M$ element vector containing ones. As only (11) has to be satisfied, the dimension $L$ can be selected arbitrarily with $L \leq M-1$. The maximum value $M-1$ corresponds to the fully adaptive GSC. When a large number of

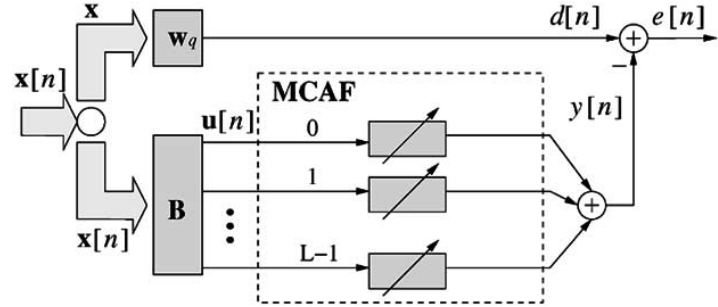

Fig. 2. General structure of the GSC, where unconstrained adaptive optimization is performed by a multichannel adaptive filter (MCAF).

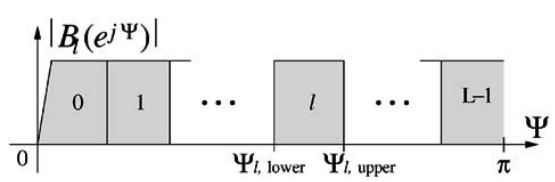

Fig. 3. Characteristics of the $L$ spatial filters contained in $\boldsymbol{B}$.

sensors is employed, we can opt for a smaller value of $L$, i.e., $L<M-1$, resulting in a partially adaptive GSC [3], which has a reduced number of DOFs and offers reduced complexity traded off against a generally somewhat inferior performance. In Section III, we will trade the loss of DOFs against a specific design of the blocking matrix.

\section{B. Partially Adaptive Subband-Selective GSC}

Consider a unity amplitude complex input wave with angular frequency $\omega$ and DOA $\theta$. Referring to Fig. 1, the waveform impinges with a time delay $\Delta \tau$ on adjacent sensors separated by $d$ in a medium with propagation speed $c$. The received phase vector at the sensor array, $\underline{x}$, is

$$
\underline{x}=\left[1 e^{-j \omega \Delta \tau} \cdots e^{-j \omega(M-1) \Delta \tau}\right]^{T} \quad \text { with } \quad \Delta \tau=\frac{d}{c} \sin \theta .
$$

Assume that the array sensors are spaced by half a wavelength of the maximum signal frequency and the temporal sampling frequency $\omega_{s}$ is twice the maximum signal frequency, i.e., $d=$ $\lambda_{s}=c T_{s}$, where $T_{s}$ is the temporal sampling period; then, we get $\Delta \tau=T_{s} \sin \theta$. Noting that $\omega T_{s}=\Omega$, where $\Omega$ is the normalized angular frequency of the signal, the phase vector can be written as

$$
\underline{x}=\left[\begin{array}{llll}
1 & e^{-j \Omega \sin \theta} & \cdots & e^{-j(M-1) \Omega \sin \theta}
\end{array}\right]^{T} .
$$

Using the substitution $\Psi=\Omega \sin \theta$, the $l$ th output of the blocking matrix, $u_{l}[n], l=0, \ldots, L-1$, can be denoted as

$$
\begin{aligned}
u_{l}[n] & =\boldsymbol{b}_{l}^{H} \cdot \underline{\boldsymbol{x}} \cdot e^{j n \Omega}=\sum_{m=0}^{M-1} b_{l}[m] \cdot e^{-j m \Psi} \cdot e^{j n \Omega} \\
& =B_{l}\left(e^{j \Psi}\right) \cdot e^{j n \Omega}
\end{aligned}
$$

with $B_{l}\left(e^{j \Psi}\right) \circ \quad \bullet_{l}[m]$ being a Fourier transform pair.

When the beamformer is constrained to receive the signal of interest from broadside, the blocking matrix has to suppress any signal impinging from $\theta=0$. Therefore, at $\Psi=0$ the response of $B_{l}\left(e^{j \Psi}\right)$ has to be zero, which has been indicated in (11). Now, we arrange $B_{l}\left(e^{j \Psi}\right), l=0, \ldots, L-1$, over the interval $\Psi \in[0 ; \pi]$ as shown in Fig. 3, satisfying

$$
\left|B_{l}\left(e^{j \Psi}\right)\right|= \begin{cases}1, & \text { for } \Psi \in\left[\Psi_{l, \text { lower }} ; \Psi_{l, \text { upper }}\right] \\ 0, & \text { otherwise. }\end{cases}
$$




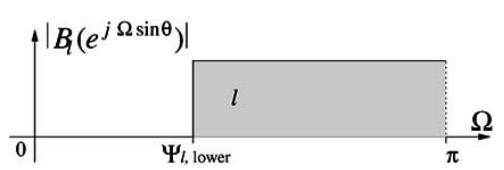

Fig. 4. Temporal filtering effect of the $l$ th spatial filter in $\boldsymbol{B}$.

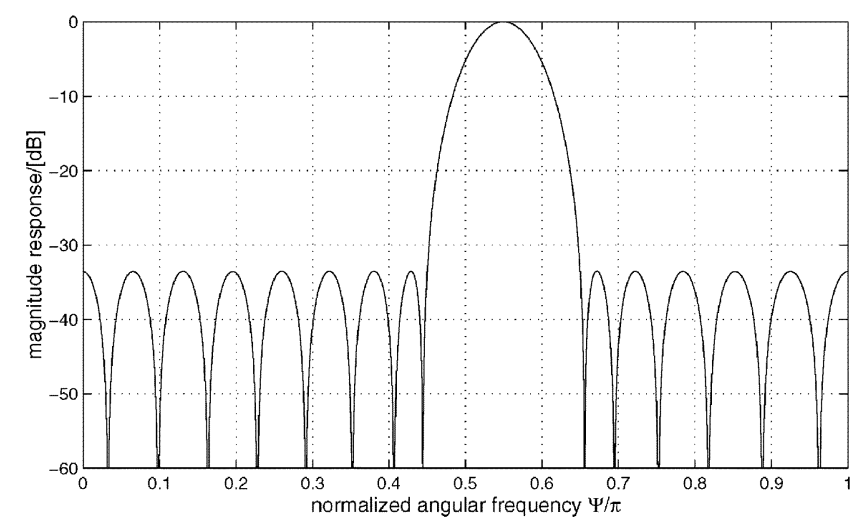

Fig. 5. Frequency response of a bandpass filter as an example for the column vectors of $\boldsymbol{B}$.

As $|\sin \theta| \in[0 ; 1]$ when $\theta \in[-\pi / 2 ; \pi / 2]$, the possible maximum frequency component of the $l$ th output $u_{l}[n]$ is $\Omega=\pi$, which corresponds to $|\sin \theta|=\Psi_{l \text {,upper }} / \pi$, while the possible minimum frequency component is $\Omega=\Psi_{l \text {,lower }}$, which corresponds to $|\sin \theta|=1$. Then, we have the response of $B_{l}\left(e^{j \Psi}\right)$ as a function of $\Omega$ in the following:

$$
\left|B_{l}\left(e^{j \Omega \sin \theta}\right)\right|= \begin{cases}1, & \text { for } \Omega \in\left[\Psi_{l, \text { lower }} ; \pi\right] \\ 0, & \text { otherwise }\end{cases}
$$

as shown in Fig. 4.

As a simple example to show this effect, Fig. 5 depicts the frequency response of a 30-tap bandpass filter designed by the MATLAB function remez [13]. Its magnitude response with respect to signals of different frequencies and DOAs is shown in Fig. 6. To see its highpass filtering effect more clearly, the magnitude response is drawn over frequency only in Fig. 7, yielding an ensemble of curves for the various DOA $\theta \in[-\pi / 2 ; \pi / 2]$.

Thus, the blocking matrix not only decomposes the received signals and interferences in the spatial domain, but also in the temporal domain, where the column vectors of $B$ simultaneously perform a temporal high-pass filtering operation. With increasing $l$, these filters are associated with a tighter and tighter highpass spectrum and the last output $(L-1)$ only contains the ultimate highpass component. Thus, if we decompose each of these highpass signals into subbands in a similar way as in [7] and [14], the subband signals residing in the corresponding lowpass subbands will be zero and can be omitted from the subsequent subband adaptive processing. This subband setup is shown in Fig. 8. The blocks labeled $A$ perform analysis operations, splitting the signal into $K$ frequency bands by means of a $K$-channel filter bank with decimation ratio $N$. Within each subband, an independent unconstrained multichannel adaptive filter is operated, and a synthesis filter bank, labeled $S$, recombines the different subsystem outputs to the full-band output $e[n]$.

Let us now analyze the computational complexity of the above system. The adaptation in subbands requires only approximately
$K / N^{2}$ (or $K / N^{3}$ ) of the operations required for a full-band adaptive algorithm with a complexity of $\mathcal{O}\left(L_{a}\right)$ (or $\mathcal{O}\left(L_{a}^{2}\right)$ ), where $L_{a}$ is the total number of coefficients in the full-band realization [7]. If sufficiently selective column vectors $\boldsymbol{b}_{l}$ can be designed, the first $(k=0)$ MCAF would be a single channel adaptive filter drawing its low-frequency input solely from the first branch of $\boldsymbol{B}$. The second $(k=1)$ MCAF block in Fig. 8 will most likely only cover some of the lower branches of $\boldsymbol{B}$, while finally only the last MCAF $(K-1)$ consists of all of the $L$ nonsparse channels. Thus, under ideal conditions, on average the dimensionality of the MCAFs can be reduced by half, with a proportional decrease in computational complexity. Considering the whole subband-selective GSC, only $L K / 2(M-1) N^{2}$ (or $\left.L K / 2(M-1) N^{3}\right)$ of the computations of the traditional fully adaptive GSC schemes are required.

As an example for discarding channels in the MCAF blocks, assume a scenario with real valued sensor signals and a blocking matrix with $L=4$ columns, whereby the frequency responses $B_{l}\left(e^{j \Psi}\right)$ of the column vectors are ideal as given in Fig. 9(a). By employing eight-channel $(K=8)$ oversampled generalized DFT (GDFT) filter banks [15], [16] with frequency responses shown in Fig. 9(b), each of the blocking matrix outputs $u_{l}[n]$, $l=0,1,2,3$, is decomposed into eight subband signals $u_{l, k}$, $k=0,1, \ldots, 7$. They form, in total, eight subband MCAF blocks, as shown in Fig. 10. Since the input signals are real, we only need to process the first four subband MCAF blocks. From the characteristics of the frequency responses in Fig. 9(a) and (b), it can be seen that some subband channels will have zero power and can be discarded. The pattern for discarding these channels is given in Fig. 10, where the discarded subbands in the last four subband MCAF blocks are also shown for completeness. With increasing $L$ and $K$, the number of discarded subband channels becomes approximately half of the total number of subbands. However, in practice, the frequency responses of the column vectors and the filter banks are not ideal and finite transition bands are unavoidable, such that the number of discarded subbands will be smaller than in the ideal situation.

\section{Cosine-Modulated Blocking Matrix}

In our subband-selective GSC, the blocking matrix plays a central role and a column vector design with a good band-selective property is of great importance. We may design each of the column vectors independently subject to the constraint in (11). In order to reduce the design and implementation complexity of the blocking matrix, here we propose a cosine-modulated version, where all the column vectors are derived from a prototype vector by cosine-modulation, which is a widely used method in the implementation of filter banks [17]. The broadside constraint can be guaranteed by imposing zeros appropriately on the prototype vector.

Assume that the prototype vector is $h[m], m=0, \ldots, M-1$. It is shifted along the frequency axis by $(2 l+3) \pi / 2 L+2$ and $-(2 l+3) \pi / 2 L+2$ using type-IV cosine modulation [17] to obtain the $l$ th column vector $b_{l}[m], l=0, \ldots, L-1$

$$
\begin{aligned}
& b_{l}[m]=h[m] \\
& \quad \cdot \cos \left[\frac{\pi}{2 L+2}(2 l+3)\left(m-\frac{M-1}{2}\right)-(-1)^{l} \frac{\pi}{4}\right] .
\end{aligned}
$$




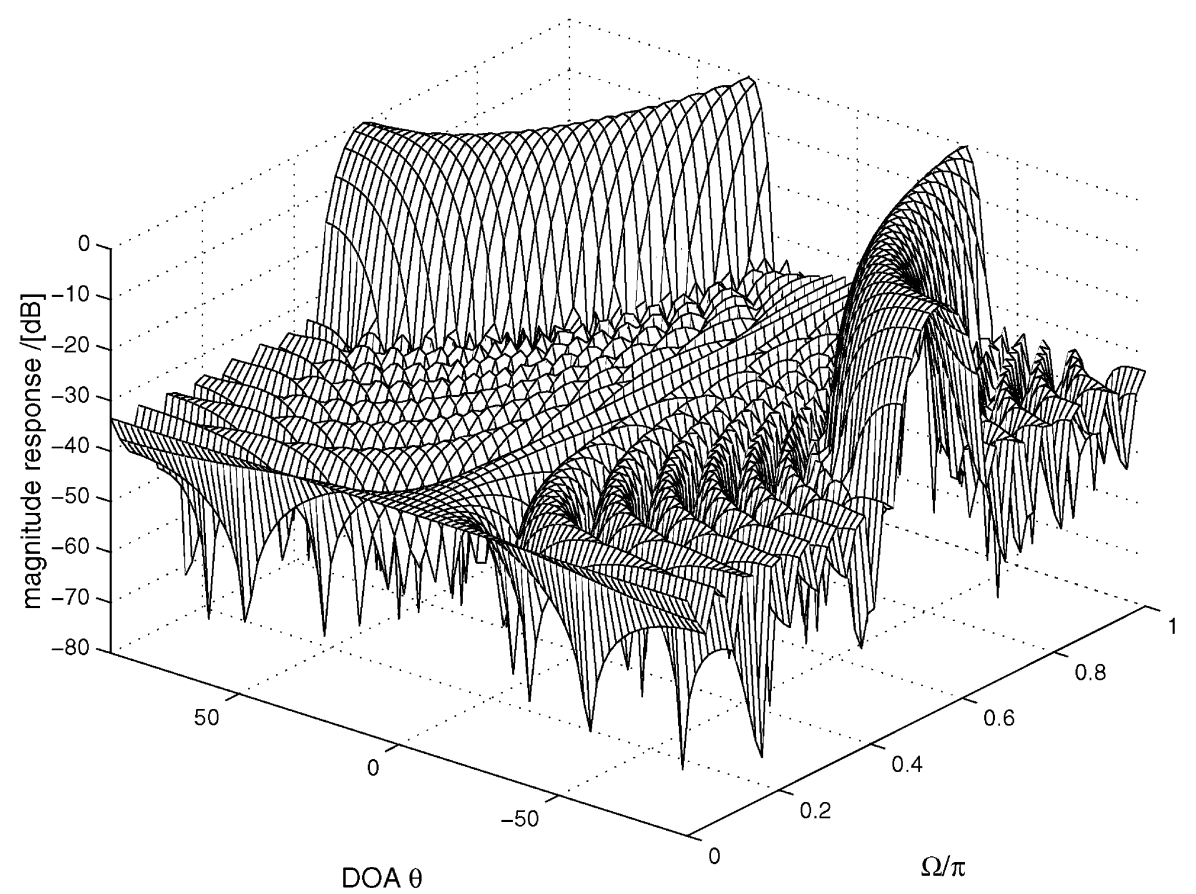

Fig. 6. Three-dimensional magnitude response of a bandpass filter to signals with different frequencies and DOAs.

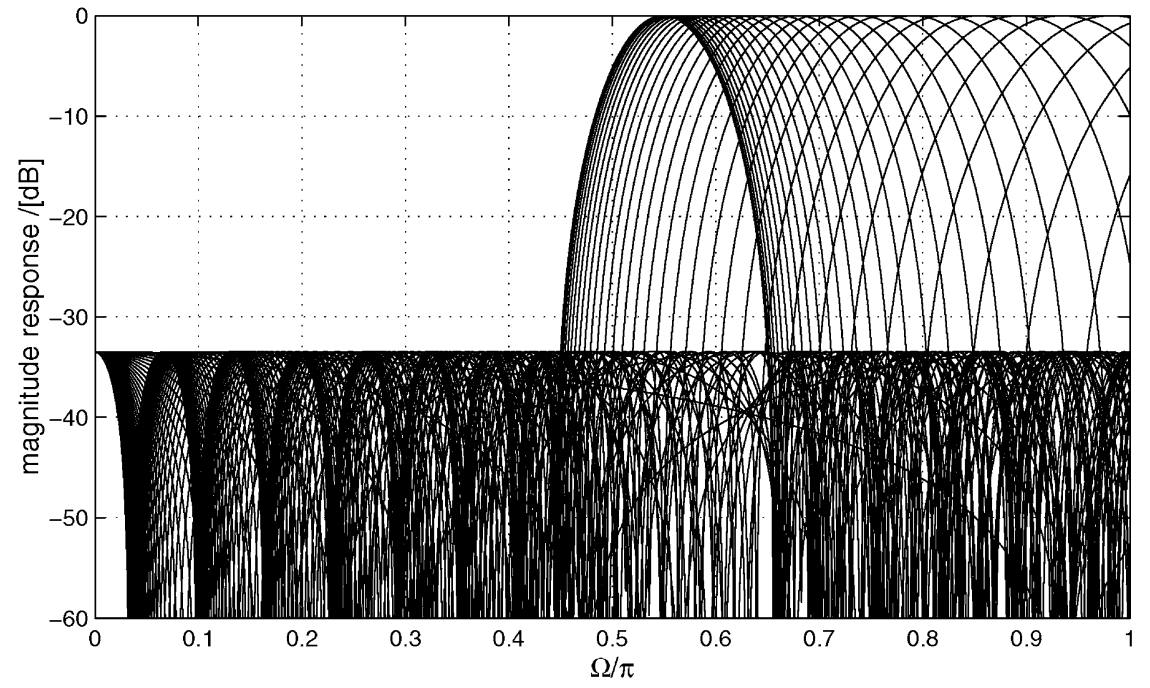

Fig. 7. Response of a bandpass filter to signals with different frequencies as a column vector of the blocking matrix $\boldsymbol{B}$.

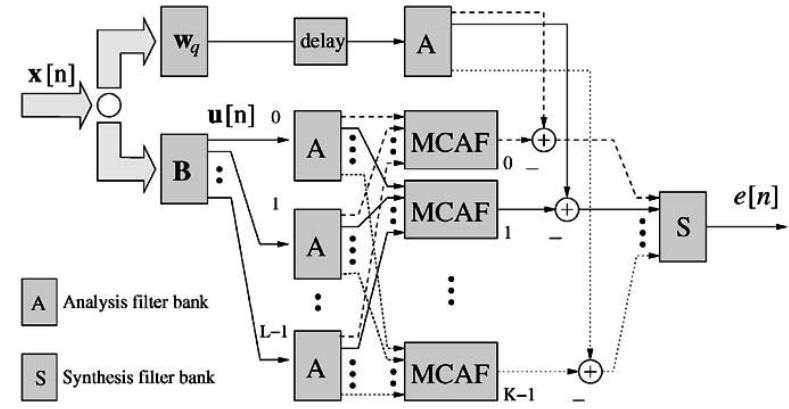

Fig. 8. Subband decomposition applied to the output of the blocking matrix. Independent MCAF (as defined in Fig. 2) is applied to each subband.

In fact, these column vectors constitute the last $L$ analysis filters in an $(L+1)$-channel maximally decimated cosine-modulated filter bank system. If the polyphase matrix of the analysis (a) $\left|B_{l}\left(e^{j \Psi}\right)\right|$

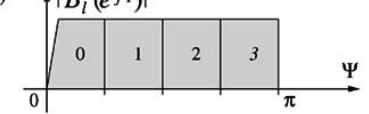

(b)

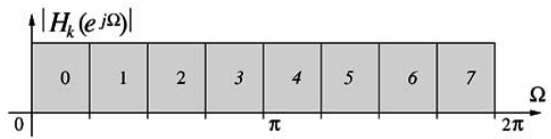

Fig. 9. (a) Frequency responses of the four-column vectors $B_{l}\left(e^{j \Psi}\right)$, $l=0,1,2,3$. (b) Frequency responses of the eight-channel analysis filter bank $H_{k}\left(e^{j \Omega}\right), k=0, \ldots, 7$.

filter bank is paraunitary, then this filter bank system is perfect reconstructing, and the column vectors are thus orthogonal to each other [17]. Here, orthogonality is not required; however, the column vectors $b_{l}[m]$ have to exhibit a good band-selection property and fulfill the broadside constraint $\left.B_{l}\left(e^{j \Psi}\right)\right|_{\Psi=0}=0$. 


\begin{tabular}{|c|c|c|c|c|c|c|c|}
\hline$u_{3,0}$ & $u_{3,1}$ & $u_{3,2}$ & $u_{3,3}$ & $u_{3,4}$ & $u_{3,5}$ & $u_{3,6}$ & $u_{3,7}$ \\
\hline$u_{2,0}$ & $u_{2,1}$ & $u_{2,2}$ & $u_{2,3}$ & $u_{2,4}$ & $u_{2,5}$ & $u_{2,6}$ & $u_{2,7}$ \\
\hline$u_{1,0}$ & $u_{1,1}$ & $u_{1,2}$ & $u_{1,3}$ & $u_{1,4}$ & $u_{1,5}$ & $u_{1,6}$ & $u_{1,7}$ \\
\hline$u_{0,0}$ & $u_{0,1}$ & $u_{0,2}$ & $u_{0,3}$ & $u_{0,4}$ & $u_{0,5}$ & $u_{0,6}$ & $u_{0,7}$ \\
\hline
\end{tabular}

Fig. 10. Components of the subband MCAF blocks (where only shaded squares will be processed; remaining unshaded subband channels are discarded).

To ensure the zero response $\left.B_{l}\left(e^{j \Psi}\right)\right|_{\Psi=0}=0$ after modulation, the frequency response $H(z)\left(z=e^{j \Psi}\right)$ of the prototype vector $h[\mathrm{~m}]$ should have one zero at each point of $\Psi_{l}=$ $\pm(2 l+3) \pi / 2 L+2, l=0, \ldots, L-1$. If we factorize $H(z)$ into two parts according to

$$
H(z)=P(z) Q(z)
$$

with

$$
\begin{aligned}
Q(z)= & \prod_{l=0}^{L-1}\left(1-e^{j(2 l+3 / 2 L+2) \pi} z^{-1}\right) \\
& \times\left(1-e^{-j(2 l+3 / 2 L+2) \pi} z^{-1}\right)
\end{aligned}
$$

then the broadside constraint will be automatically satisfied for all the column vectors and the free parameters contained in $P(z)$ can be used to optimize its frequency response. By this factorization, the design of the blocking matrix becomes an unconstrained optimization problem of the prototype vector. The objective function we minimize is

$$
\Phi=\int_{\Psi_{s}}^{\pi}\left\|H\left(e^{j \Psi}\right)\right\|^{2} d \Psi
$$

where $\Psi_{s}$ is the stop-band edge. To solve this unconstrained optimization problem, we employ here the subroutines BCONF/DBCONF of the IMSL library [18]. A design example for the blocking matrix with $M=28$ sensors and $L=11$ column vectors is given in Fig. 11.

In fact, the constraint in (11) can be regarded as the zero-order derivative constraint [12]. If $(S-1)$-order derivative constraints [12] are employed for the GSC, we can replace $Q(z)$ in (18) by $Q(z)^{S}$. Note however that too many DOFs might be sacrificed and a satisfying performance may not be achieved for smallscale arrays.

\section{Simulations AND Results}

The following simulation is based on a beamformer setup with $M=21$ sensors and $J=90$ taps for each attached sensor filter. The signal of interest illuminates the array from broadside and two interfering signals impinge from $\theta=20^{\circ}$ and $\theta=-40^{\circ}$, respectively, both of which cover the frequency band $\Omega=[0.30 \pi ; 0.70 \pi]$ with a signal-to-interference

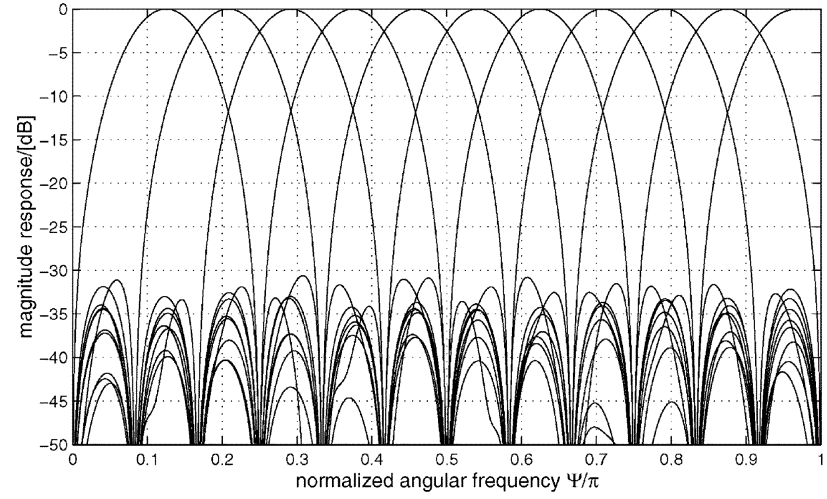

Fig. 11. A design example for a $28 \times 11$ blocking matrix.

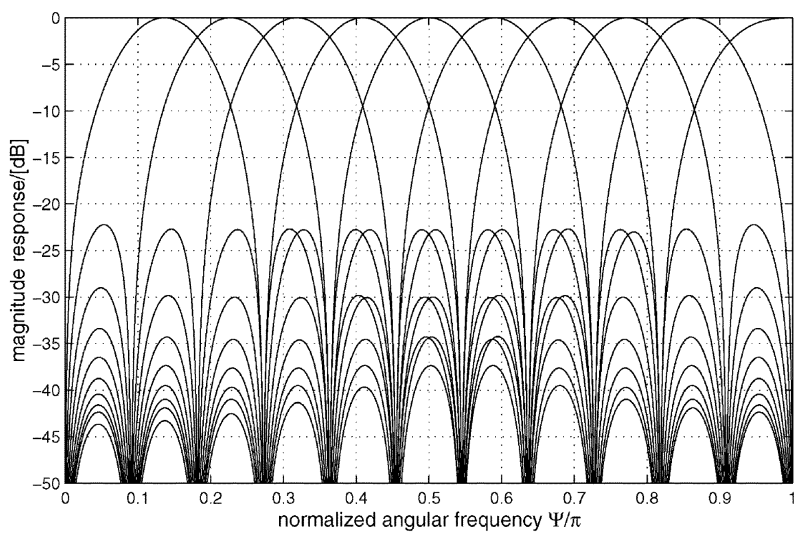

Fig. 12. Frequency responses of the $21 \times 10$ blocking matrix.

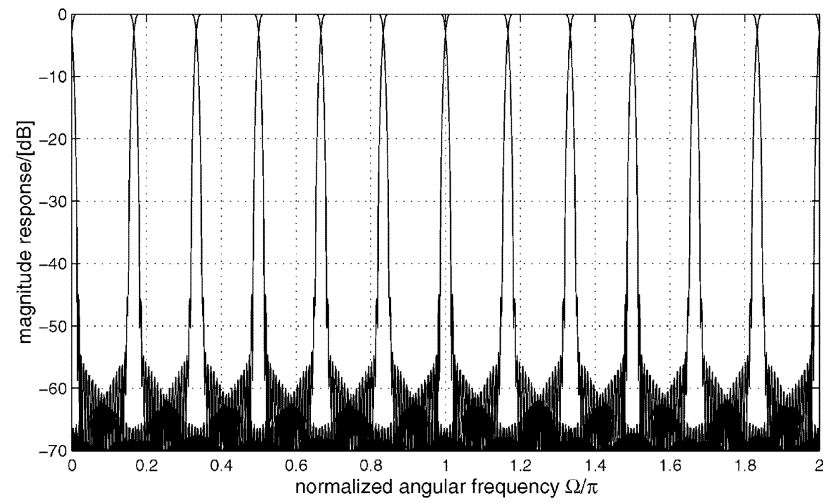

Fig. 13. Frequency responses of the $K=12$ channel filter banks decimated by $N=10$.

ratio (SIR) of $-30 \mathrm{~dB}$. Additionally, all sensors receive spectrally and spatially uncorrelated noise at $20-\mathrm{dB}$ signal-to-noise (SNR).

In our subband-selective GSC, the dimension of $\boldsymbol{B}$ is $21 \times 10$ $(L=10)$, with its characteristic shown in Fig. 12. Each of the blocking matrix outputs and the reference signal $d[n]$ are divided into $K=12$ subbands by oversampled GDFT filter banks [15], [16] with decimation ratio $N=10$. The frequency responses of the analysis filters, which are based on a prototype filter with 240 coefficients, are depicted in Fig. 13. The adaptive 


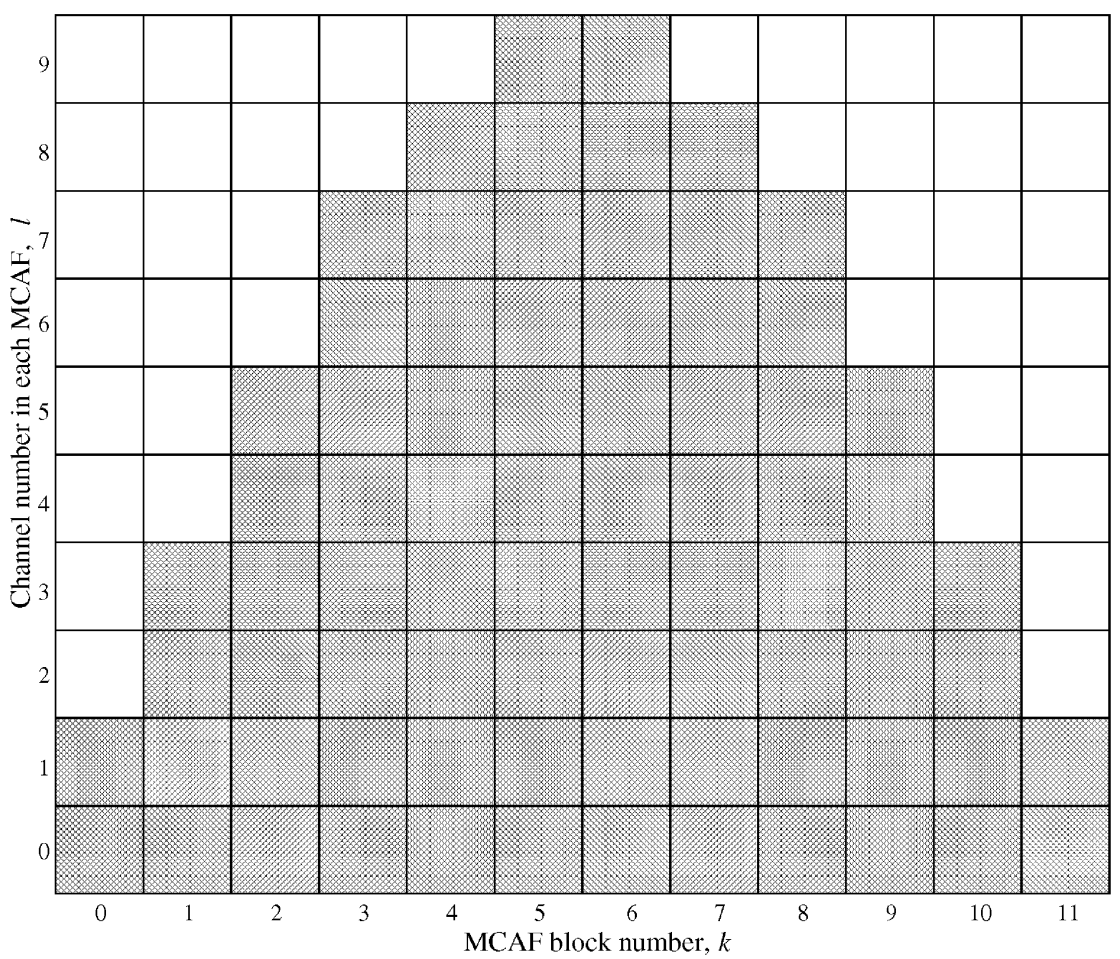

Fig. 14. Channels discarded in each MCAF block in our simulation (where only shaded squares will be processed; remaining unshaded subband channels are discarded).

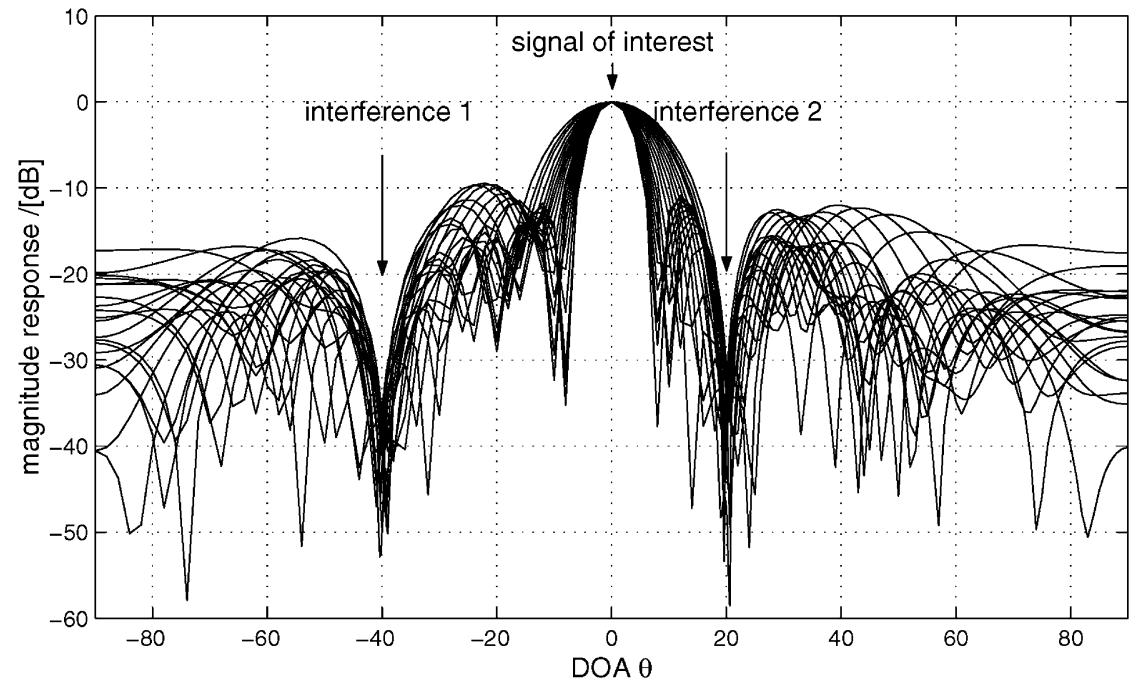

Fig. 15. Resulting beampattern for the subband-selective GSC over the frequency band $[0.3 \pi ; 0.7 \pi]$.

filter length in each subband is $J / N=9$. In each MCAF block, we discard channels with very low signal power according to Fig. 14.

The beamformer's response of the subband-selective GSC is shown in Fig. 15 over the frequency band $\Omega=[0.3 \pi ; 0.7 \pi]$. From this beampattern, we see that the subband-selective GSC is capable of suppressing the interference effectively by forming appropriate nulls in the directions of interference.

Fig. 16 shows the learning curves of a normalized LMS (NLMS) algorithm with a step size of 0.3. The depicted performance measure is the mean square value of the residual error, i.e., the beamformer output $e[n]$ minus the appropriately delayed signal of interest. The convergence rate of the subband-selective GSC is significantly improved over the traditional fully adaptive full-band GSC due to the combined decorrelation effect of both the blocking matrix and the filter banks. This decorrelation is performed in the temporal domain by the filter banks and in both the temporal and spatial domains by the blocking matrix, as shown by (15) and (16). In terms of computational complexity, the proposed subband-selective GSC only needs $37 \%$ multiplications of its full-band counterpart. 


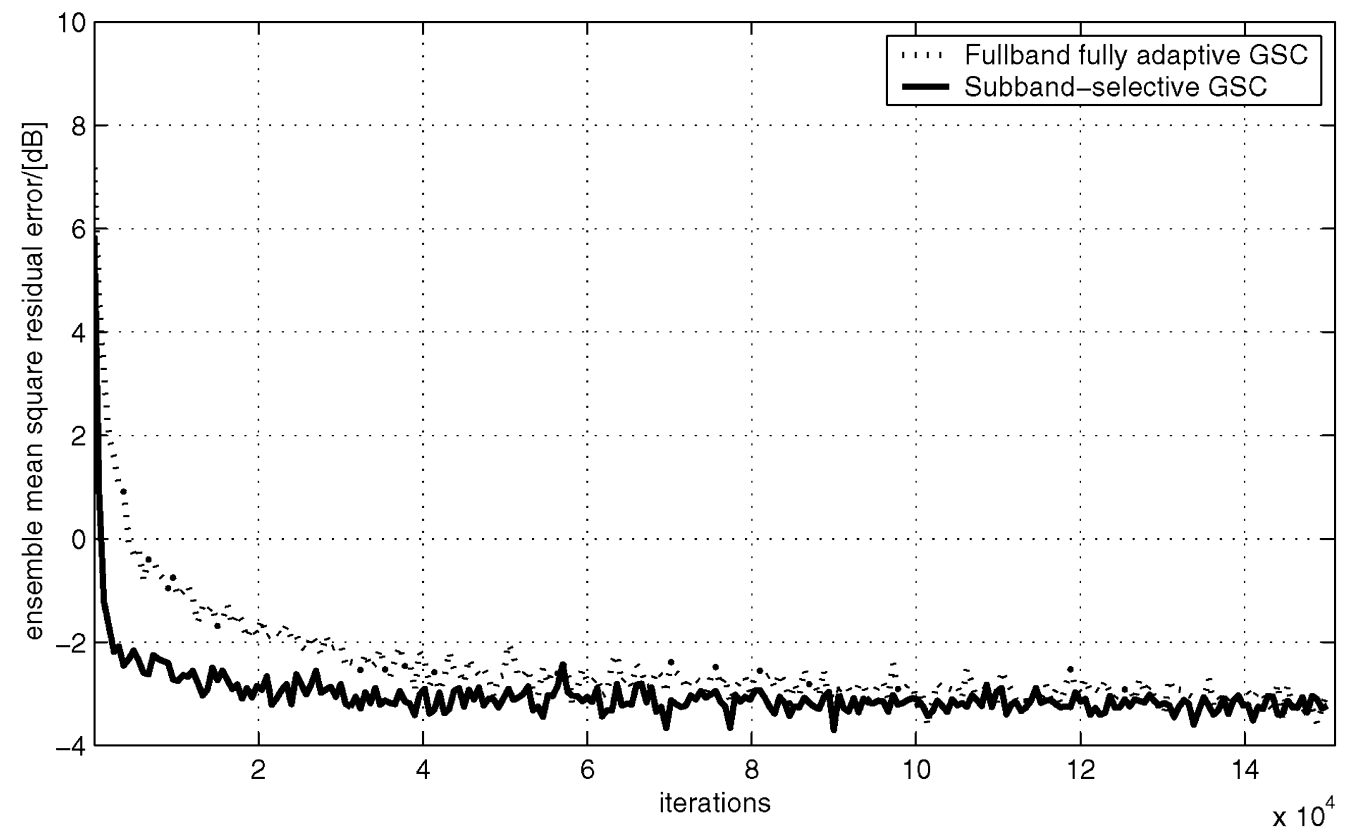

Fig. 16. Learning curves of a full-band fully adaptive GSC and the proposed subband-selective GSC.

\section{CONCLUSION}

A spatially/spectrally subband-selective GSC for partially adaptive broadband beamforming with cosine-modulated blocking matrix has been proposed. In this structure, the column vectors of the blocking matrix are derived from a prototype vector by cosine-modulation and these vectors constitute a series of bandpass filters, which decompose the impinging signals into components of specific DOAs and frequencies and lead to band-limited spectra of the blocking matrix outputs. Subband methods were employed to remove the associated redundancy by discarding the corresponding lowpass subbands. The combination of partial adaptation, subband decomposition, and discarding permits a considerably reduced computational complexity. As demonstrated by our simulations, the proposed approach also has the additional benefit of faster convergence for LMS-type adaptive algorithms.

\section{REFERENCES}

[1] H. L. Van Trees, Optimum Array Processing, Part IV of Detection, Estimation, and Modulation Theory. New York: Wiley, 2002.

[2] D. J. Chapman, "Partial adaptivity for large arrays," IEEE Trans. Antennas Propagat., vol. 24, pp. 685-696, Sept. 1976.

[3] B. D. Van Veen and R. A. Roberts, "Partially adaptive beamforming design via output power minimization," IEEE Trans. Acoust., Speech, Signal Processing, vol. 35, pp. 1524-1532, 1987.

[4] H. Yang and M. A. Ingram, "Design of partially adaptive arrays using the singular-value decompostion," IEEE Trans. Antennas Propagat., vol. 45, pp. 843-850, May 1997.

[5] Y. Y. Wang and W. H. Fang, "Wavelet-based broadband beamformers with dynamic subband selection," IEICE Trans Commun., vol. E83B, no. 4, pp. 819-826, Apr. 2000.

[6] S. Weiss, R. W. Stewart, M. Schabert, I. K. Proudler, and M. W. Hoffman, "An efficient scheme for broadband adaptive beamforming," in Asilomar Conf. Signals, Systems, Computers, vol. I, Monterey, CA, Nov. 1999, pp. 496-500.

[7] W. Liu, S. Weiss, and L. Hanzo, "Subband adaptive generalized sidelobe canceller for broadband beamforming," in Proc. IEEE Workshop Statistical Signal Processing, Singapore, Aug. 2001, pp. 591-594.
[8] W. H. Neo and B. Farhang-Boroujeny, "Robust microphone arrays using subband adaptive filters," IEE Proc.-Vision, Image Signal Processing, vol. 149, no. 1, pp. 17-25, Feb. 2002.

[9] L. J. Griffith and C. W. Jim, "An alternative approach to linearly constrained adaptive beamforming," IEEE Trans. Antennas Propagat., vol. 30, pp. 27-34, Jan. 1982.

[10] O. L. Frost III, "An algorithm for linearly constrained adaptive array processing," Proc. IEEE, vol. 60, pp. 926-935, Aug. 1972.

[11] S. Haykin, Adaptive Filter Theory, 3rd ed. Englewood Cliffs, NJ: Prentice Hall, 1996.

[12] K. M. Buckley and L. J. Griffith, "An adaptive generalized sidelobe canceller with derivative constraints," IEEE Trans. Antennas Propagat., vol. 34, pp. 311-319, Mar. 1986.

[13] Matlab 6.1. Natick, MA: The Mathworks, Inc., 2001.

[14] W. Liu, S. Weiss, and L. Hanzo, "A novel method for partially adaptive broadband beamforming," in Proc. IEEE Workshop Signal Processing Systems, Antwerp, Belgium, Sept. 2001, pp. 361-372.

[15] M. Harteneck, S. Weiss, and R. W. Stewart, "Design of near perfect reconstruction oversampled filter banks for subband adaptive filters," IEEE Trans. Circuits Syst. II, vol. 46, pp. 1081-1086, Aug. 1999.

[16] S. Weiss and R. W. Stewart, "Fast implementation of oversampled modulated filter banks," IEE Electron. Lett., vol. 36, no. 17, pp. 1502-1503, Aug. 2000.

[17] P. P. Vaidyanathan, Multirate Systems and Filter Banks. Englewood Cliffs, NJ: Prentice Hall, 1993.

[18] IMSL Fortran Numerical Libraries. San Ramon, CA: Visual Numerics, Inc., 2002.

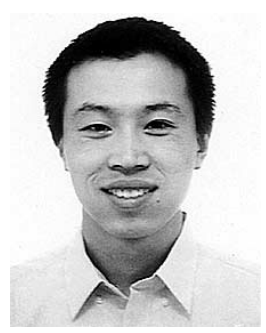

Wei Liu (S'01-M'04) was born in Hebei, China, on January 22,1974 . He received the the B.Sc. degree in space physics with a minor in electronics and the L.L.B. degree in intellectual property law from Peking University, Beijing, China, in 1997 and 1996, respectively, the M.Phil. degree from the Department of Electrical and Electronic Engineering, University of Hong Kong, Hong Kong, China, in 2000, and the $\mathrm{Ph} . \mathrm{D}$. degree in computer science from the School of Electronics and Computer Science, University of Southampton, Southampton, U.K., 2003.

Currently, he is a Research Fellow at the Communications Research Group, University of Southampton. His research interests include filter banks and wavelets, adaptive and array signal processing. 


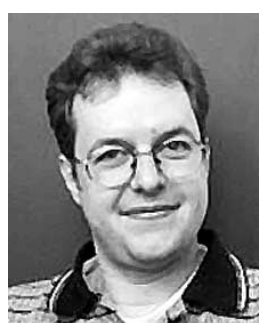

Stephan Weiss (''97-A'98-M'99) received the Dipl.-Ing. degree from the University of Erlangen-Nürnberg, Erlangen, Germany, in 1995 and the Ph.D. degree from the University of Strathclyde, Glasgow, U.K., in 1998, both in electronic and electrical engineering.

Since 1999, he has been a Lecturer in the Communications Group at the University of Southampton, Southampton, U.K., prior to which he held a Visiting Lectureship at the University of Strathclyde. In 1996 and 1997 he was a Visiting Scholar at the University of Southern California. His research interests include adaptive filtering, multirate systems, and signal expansions, with applications in audio, communications, and biomedical signal processing. He has authored and coauthored more than 60 technical publications, including a book.

Dr. Weiss was a co-recipient of the 2001 Annual Research Award of the German Hearing Aid Society. He is a Member of the VDE.

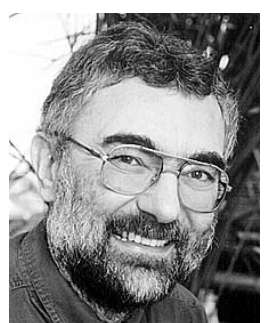

Lajos Hanzo (M'91-SM'92-F'04) received the B.S. degree in electronics in 1976 and the Ph.D. degree in 1983.

During his career in telecommunications he has held various research and academic posts in Hungary, Germany, and the U.K. Since 1986, he has been with the Department of Electronics and Computer Science, University of Southampton, Southampton, U.K., where he holds the chair in telecommunications. He has coauthored ten books on mobile radio communications and about 450 research papers. Currently, he heads an academic research team, working on a range of research projects in the field of wireless multimedia communications sponsored by industry, the Engineering and Physical Sciences Research Council (EPSRC) U.K., the European 1ST Programme, and the Mobile Virtual Centre of Excellence (VCE), U.K.

Dr. Hanzo has organized and chaired conference sessions, presented overview lectures, and has been awarded a number of distinctions. He is an IEEE Distinguished Lecturer. 